Local amputation of the breast in young women produces a very unsatisfactory state of affairs. The patients become introspective, worry a lot, and tend to become chronic invalids. Many drift to the out-patient departments of the neurological hospitals. I have case reports of 20 unmarried women under 30 years of age who have been subjected to local removal of one or both breasts because of a history of a blood-stained discharge from the nipple. These women are afraid to marry because they consider that they are not normal. This state of affairs must not be allowed to continue, as it means a life of constant misery.

The following up of operation cases is most important if we are to get a clear idea of the real value of conservative surgery. Of my 52 cases of duct papilloma of the breast I have

follow-up" records of 35 ; six patients are abroad and I cannot trace them. However, the clinical history of three cases will show the real advantage of conservative surgery for duct papilloma.

\section{Case Histories}

Case 1.-A nurse, aged 24, who had just finished her training in a London hospital, was seen in July, 1915, with a history of a blood-stained discharge from the left breast for some nine months. Nothing could be felt on examination and no discharge was visible from the nipple. The patient was told to come and see me when there was any further discharge from the nipple. In Sept., 1915, she came again, and a small cystic lump could be felt just lateral to the nipple in the lower and inner quadrant of the breast. A slight blood-stained discharge could $\cdot$ be expressed from the nipple. Operation was undertaken, and a small intracystic papilloma was excised. The papilloma had a definite stalk, and microscopically was quite innocent. The patient left hospital a week later. She was seen in 1919 before her marriage, as she was concerned about the effect of pregnancy on the breast. The breast was quite normal, and there had been no discharge from the nipple since the operation. The patient was reassured that no ill effects would supervene if pregnancy took place. She was seen again in 1936 because of cholecystitis due to gall-stones. She had five children-three boys and two girls - and had breast-fed all of them without any difficulty whatever.. Cholecystectomy was carried out in July, 1936, and recovery was uneventful.

Case 2.-A married woman aged 32 was seen in Jan., 1920; she was sent to me to have her right breast removed because of an intermittent blood-stained discharge. She was a big woman with large firm breasts. A small lump the size of a pea could be felt in the lower and outer quadrant of the breast. She was prepared to have the breast removed, but was advised to undergo excision of the lump instead. Local excision of the papilloma was carried out and the growth was found to be innocent. The patient was last seen in 1939, when she was in good health; the breast was quite normal, although somewhat atrophic. Her periods had stopped some three years previously.

Case 3.-An unmarried woman, aged 26 , a well-known golfer, was seen in 1922 because of a blood-stained discharge from the left nipple. There was little to make out on clinical examination, and the patient was told not to worry and to forget about her breast. She was seen in 1923 and again in 1924, and on neither occasion could anything be felt in the breast, although there was a slight intermittent discharge of blood-stained fluid from the nipple. When seen in May, 1925, a small lump could be felt in the lower part of the breast just below the nipple. This was excised, and proved to be a simple papilloma. Six months later the patient married and was not seen again till 1932, when her child, aged 6 , was operated upon for appendicitis. Her breasts were then quite normal and there had been no discharge from the nipple since the operation. She had not breast-fed her child, as her doctor thought it might cause trouble in the breast. This idea, which still prevails in some quarters, is quite erroneous.

\section{Conclusion}

These three cases clearly demonstrate the value of conservative surgery in the treatment of duct papilloma of the breast. Every hospital of any size has specimens of small innocent papillomata because of which the breast had been removed. Conservative surgery will not provide such nice museum specimens, but it will produce contented and very thankful patients.

B. Levant (J. Urol., 1944, 52, 63) records a fatal case of sarcoma of the penis in a negro aged 29 . Deep $x$-ray therapy was beneficial. Biopsy from an inguinal gland, though confirmatory of metastases, hastened the spread of the tumour, with general systemic failure.

\section{"NEUTRAL PROFLAVINE SULPHATE" (MONOSULPHATE OF 2 : 8-DIAMINO- ACRIDINE) \\ ITS LOCAL ACTION IN INFECTED WOUNDS INVOLVING BONE \\ BY}

\author{
J. F. HEGGIE, M.B., B.Sc.Glas.
}

Surg. Cmdr., R.N.V.R.; Bacteriological Specialist,

\section{G. B. R. WARNOCK, M.B., M.R.C.P.Ed. \\ Capt., R.A.M.C.; Graded Pathologist} AND

R. W. NEVIN, M.B., F.R.C.S.

Lieut.-Col., R.A.M.C.; Officer i/c Surgical Division

\section{(From a General Hospital)}

It has been realized for a considerable time that the high acidity of proflavine suiphate B.P. is a serious drawback, and various methods have been suggested for countering this, such as a preliminary neutralization with sodium bicarbonate or the addition of a buffering salt. There is now available, however, "neutral proflavine sulphate," which possesses all the activity of the B.P. salt and yet gives a neutral solution. The relationship of neutral proflavine sulphate to proflavine sulphate B.P. may be compared with that of quinine sulphate to quinine bisulphate-i.e., the neutral sulphate has one equivalent of acid, while the B.P. salt contains two equivalents. In a $0.1 \%$ solution neutral proflavine sulphate is stated to have a $p \mathrm{H}$ of 6.5 , as contrasted with $p \mathrm{H} 2.5$ of proflavine sulphate B.P. Our brief experience with a sample of neutral proflavine sulphate indicates, however, that its action towards the tissues is the same as that of proflavine sulphate B.P., and consequently, like it, should be used (1) in small doses of from 0.2 to $0.5 \mathrm{~g}$. in discharging wounds, and (2) diluted to $0.5 \%$ in sulphathiazole powder for local application to wounds subjected to fresh débridement. We would also suggest that the optimum concentration of proflavine sulphate B.P. in sulphathiazole-proflavine powder is $0.2 \%$, rather than the strength of $1 \%$ at present employed prophylactically in " debrided" wounds.

The cases treated were with us for only a short time. They comprised gunshot and similar wounds with compound fractures (often comminuted) which had been infected and relatively untreated for from six months to two years. The majority had had repeated sequestrectomies, with, in some instances, sulphonamides locally and/or orally in support. By the time of our observations general nutrition was restored; none of the patients were more than slightly anaemic (less than $80 \% \mathrm{Hb}$ ). Neutral proflavine sulphate powder, sterilized as are the sulphonamides, was applied to the wound area at the time of the last sequestrectomy and the wound lightly packed. In a few cases it was applied 2 to 14 days after sequestrectomy, and in others it was inserted into sinuses from the depths of which sequestra had been removed earlier. Thus the powder was applied to surfaces with granulations forming and established and with exudate in varying amount, as well as to wounds "debrided" afresh, with curetted and guttered bone, curetted sinuses, etc., in which no granulation tissue and little exudate or pus was present.

\section{Technique of Treatment}

At first, having regard to the relatively neutral character of neutral proflavine sulphate, doses of from 1 to $5 \mathrm{~g}$ : were applied, the former to small and the latter to large wounds. Many patients complained of some burning pain, which during the first 24 to 48 hours was accompanied by moderate pyrexia $\left(101-102^{\circ} \mathrm{F}\right.$.), partly attributable to surgical measures. Locally, the soft tissues around the wound were inflamed, and brawny oedema extended for a short distance. The immediate wound margin was of firm, rubber-like consistence. On removing the excess neutral proflavine sulphate on the 4 th, 6 th, or 10 th day the wounds were dry and the appearance of the walls everywhere up to but not involving the skin margins was that of "tanning "-superficial coagulative necrosis-to a depth of 2 to $4 \mathrm{~mm}$. This was most pronounced in freshly "debrided" wounds, which were relatively dry, and less pronounced where 
there had been some bleeding and exudation following operation; and absent, or almost so, in cases with discharging sinuses. In these latter instances the main coagulative effect of neutral proflavine sulphate had been expended on protein precipitation in the exudate-serous, sero-sanguineous, or purulent-which could thus be said to have a "sparing" effect on the subjacent tissues.

Generally, the results obtained were not good; the doses used, even in the case of the larger wounds, were much too great, and the wounds suffered an added immediate chemical inflammation of 1 to 4 days' duration. When, however, the doses employed were smaller but still twice or thrice the recommended maximum of $0.5 \mathrm{~g}$. suggested for proflavine sulphate B.P., the results were more satisfactory. The treatment of sinuses met with varied success, and, as in the case of the major wounds, this depended largely on the completeness of surgical removal or reduction of the bulk of the infection. This last is a fundamental surgical principle which scarcely need be illustrated. Nevertheless, our experience is that it is not always followed, as it does not appear to be generally understood that all chemotherapeutic treatment is but an adjuvant to surgical procedure; and whether the agent be penicillin, an amino-acridine, or a sulphonamide, the chance of success attending its use is in varying proportion to the degree of completeness of, and to the interval of delay of application after, radical surgical treatment. This notwithstanding, we had hoped that with neutral proflavine sulphate a greater latitude would be observed on account of the greater solubility of this salt in the tissue fluids; but this was not so, as the following case illustrates:

\section{Case I}

Male aged 26. Gunshot wound right leg 9 months previously. Two months after injury: osteomyelitis lower third right tibia with sequestrum and involucrum; treated in plaster. 4th to 9th month: wound opened, explored, and drained at intervals. 8th month: inner wound broke down; wound opened up, two sinuses guttered, two cavities found in tibia and levelled up; sterile neutral proflavine sulphate powder, 5 g., applied, lightly packed; light plaster cast applied.

After operation patient complained of moderate pain for 24 to 36 hours. No fever. 6th day: excess neutral proflavine sulphate removed; whole area showed " tanning" (superficial coagulative necrosis); margins slightly indurated. 22nd day: saucerized wound neutral proflavine sulphate coagulum adherent in parts; pus welled up from posterior part of tibia-another cavity probably existed. 32nd day: outer wound broke down and discharged; advisability of amputation considered. 37th day: outer wound discharge less. Patient later transferred.

In this case it is apparent that the entire extent of the diseased bone had not been defined; later, $x$-ray examination showed diffuse osteomyelitis of the lower two-thirds of the tibia. The remaining foci in the sclerotic bone had not been sterilized by the diffusion of neutral proflavine sulphate from the depot in the under aspect of the "coagulum," because either the organisms were inaccessible or the amount of dye diffused was not sufficient.

In all of the cases in which superficial coagulative necrosis or "tanning" occurred the rate of healing was definitely impaired, despite the fact that the wound was sterile when the "coagulum" remained dry. Thus in a wound which appeared likely to close completely at a reasonably early date, despite slight to moderate degrees of infection, a stationary period resulted from the damage to the superficial layers, and was followed by a period of slow recovery to the previous rate and eventual healing. This is illustrated in the following case:

\section{Case II}

Male aged 23. Gunshot wound left leg, with compound fracture of tibia and fibula of 8 months' duration. Treated in plaster cast within 14 days of injury. Five weeks after injury: sequestrum of end of upper fibular fragment removed; exuberant granulations cut away; sulphathiazole applied locally; entrance wound 4 in. by 2 in., exist wound 6 in. by 3 in. 4th month: sequestrectomy. Later, patient up and walking, foot-drop improving, wounds healing. 6th month : sequestrectomy of tibia through old wound. Two days later, on arrival of neutral proflavine sulphate, 1 to $1.5 \mathrm{~g}$. of the sterile salt was put into the open wound. During the next four days the patient complained of severe burning pain and there was oedema of the wound area; slight pyrexia. Excess neutral proflavine sulphate removed 10 days later, when appearances of "coagulation" were seen. Four weeks after sequestrectomy: healing slowly shallow eschar-covered "ulcer," 2 in. by $1 / 2$ in., remains. Six weeks after sequestrectomy: almost healed.

In the next case, despite the fact that neutral proflavine sulphate was not applied until two weeks after major surgical treatment, the discharge dried up and the wound healed, chiefly because of the completeness of the surgical procedure and the relatively limited nature of the infection in the humera head; and, to a less extent, on account of the "sparing" action of the exudate-coagulation or tissue damage being minimal despite the relatively large amount of neutral proflavine sulphate employed.

\section{Case III}

Male aged 30. Gunshot wound right shoulder, involving the shoulder-joint, with fracture of humerus and scapula, sustained one year previously. Put up in plaster spica "soon". after injury. Bony ankylosis. Discharging sinuses for many months, increasing latterly. 12th month: sequestrectomy and curettage, much soft bone removed from humeral head; tube drains; skin stitched. One week later: pain on movement, slight discharge. 2nd week: tubes removed, sterile neutral proflavine sulphate, 1 to $1.5 \mathrm{~g}$. powder, inserted into cavity in head of humerus. No great inflammatory reaction, but slightly increased discharge. 3rd week: wound dried up; appearance of "tanning" minimal. 6th week: almost healed. 8th week: healed.

It will have been observed that in none of these cases was any sulphonamide - e.g., sulphathiazole - given orally to supplement the local chemotherapeutic treatment. This is at variance with our recommendation with regard to the necessity for both oral and local administration of sulphathiazole at the time of full surgical treatment in like cases of bone infection (Heggie, Kendall, and Heggie, 1942), but in the present instance the omission was intentional, so that the value of the neutral proflavine sulphate alone might be assessed.

\section{Bacteriostatic and Bactericidal Action}

After the application of neutral proflavine sulphate powder the wounds were not necessarily always sterilized; this varied with the kind of wound, its state, the amount of discharge. and, as regards neutral proflavine sulphate, the degree of coagulation produced, and the resulting availability in solution of that salt from the depot in the coagulum. Sterility was most certain to result when application was made at the time of full surgical treatment and the "tanning" effect of neutral proflavine sulphate was distinct-that is, when the infected focus was removed and remaining surfaces were sterilized by coagulation. In other cases, slightly to moderately infected and discharging, reduction of the bacterial flora first, with sterility later, was not uncommon. This was to be expected, and is in keeping with the known slow bactericidal effect of the aminoacridines: their chief action is bacteriostatic; they limit bacterial proliferation, while leucocytic and tissue defences play their part. In wounds of the thigh or gluteal region, reinfect:on with coliform organisms occurred-and in this regard we would emphasize the need (sometimes overlooked) for attention to the marginal skin of w'ounds, and the peculiar desire of some folks to change dressings too of ten. In other wounds, infection with Proteus and Ps. pyocyanea remained: these organisms were most numerous, and Ps. pyocyanea was most persistent. Raven (1944) has reported like changes in the bacterial flora of infected wounds when using proflavine sulphate B.P.

In vitro the bacteriostatic and bactericidal actions of neutral proflavine sulphate and proflavine sulphate B.P. are practically identical, though the latter is slightly more active (next immediate tube in a series of falling dilutions). With a series of six freshly isolated strains of Staph. pyogenes and Esch. coli, and using a large inoculum -1 million in $10 \mathrm{c.cm}$. of broth-neutral proflavine sulphate was bactericidal within 24 hours, to staphylococci at 1 in 2,000 and to the coliform organisms at 1 in 4,000 . With similar inocula in broth. organisms from the series of wounds treated with neutral proflavine sulphate were killed within 24 hours by the following dilutions of that salt :

$\begin{array}{llll}\text { Ps. pyocyanea } & \ldots & \ldots & 1 / 1000 \text { and } 1 / 2000 \\ \text { Staph. pyogenes } & \ldots & 1 / 2000(1) ; 1 / 4000(4) ; 1 / 6000(1) \\ \text { Coliforms } & \ldots & \ldots & 1 / 4000(1) ; 1 / 6000(1) \\ \text { Staph. albus } & \ldots & \ldots & 1 / 12,000(2) \\ \text { Diphtheroids } & \ldots & \ldots & 1 / 12,000(2) \\ \text { Proteus forms } & \ldots & \ldots & 1 / 12,000(3) \\ \text { Str. haemolyticus } & \ldots & 1 / 12,000(1) ; 1 / 24,000 \text { (1) }\end{array}$


The efficiency of neutral proflavine sulphate can be judged easily from consideration of the following facts:

(a) The bacteriostatic effect of the proflavine salts (in serum) on Staph. aureus and $B$. coli is evident at dilutions of $1 / 200,000$ and $1 / 100,000$ respectively (Browning et al., 1917)

(b) Neutral proflavine sulphate is soluble in serum to the extent of 1 in 300 .

(c) The amino-acridines are not rendered ineffective by pus or the breakdown products of tissues.

It is obvious, however, that neutral proflavine sulphate must be available in solution to the tissues, and its concentration in the tissue fluid should be such as rapidly to effect complete bacteriostasis, without damaging the tissues even slightly.

\section{Discussion}

It is obviously important to consider the solubility of any antiseptic. Albert and Gledhill (1943) treated a specimen of 2:8-diamino-acridine with one equivalent of sulphuric acid and obtained a "neutral sulphaté" which would remain in $25 \%$ aqueous solution at $15^{\circ} \mathrm{C}$. for a week. The solubility of amino-acridines, however, as those authors point out, is easily affected by small amounts of impurities; and the purified salts do in fact usually show a decreased solubility. Our sample of neutral proflavine sulphate, which has been shown by analysis to have the correct acid-base ratio, will dissolve to the extent of 1 in 4 in warm water, but the solution begins to crystallize after 24 hours at $15^{\circ} \mathrm{C}$. The $p \mathrm{H}$ of a $0.1 \%$ solution is 6.5 , compared with $p \mathrm{H} 6$ reported by Albert.

When serum is added to neutral proflavine sulphate powder in a test-tube the powder slowly becomes moistened and syrupy, and the supernatant serum reaches dye-saturation point $\left(0.3^{\circ}\right)$ within 6 to 12 hours. When, however, serum or albumin in $4 \%$ solution is added in equal volume to a range of saline dilutions of neutral proflavine sulphate precipitation at once occurs in the $4.5 \%$ to $0.6 \%$ solutions, being most pronounced in the range $4.5 \%$ to $1 \%$, and showing proflavine crystals as well as protein precipitates in the $4.5 \%$ to $2.0 \%$ range. If fresh serum be now added to the combined precipitates or to the protein precipitate alone, the serum takes up the dye, to saturation or equilibrium, without further precipitation, and most of the dyestuffs may thus be recovered. Similar precipitation of protein occurs with saline solutions of proflavine sulphate $B . P$. in the range $0.75 \%$ (maximum solubility) to $0.25 \%$ (trace), but not in the $0.2 \%$ solution. Proflavine sulphate $B . P$. is highly acid, with a $p \mathrm{H} 2$ in $0.1 \%$ water, being extensively hydrolysed in solution; so it is possible that neutral proflavine sulphate is dissociated to some extent in saline solution and the $p \mathrm{H}$ value of the stronger neutral proflavine sulphate saline solutions is nearer the isoelectric point of plasma proteins

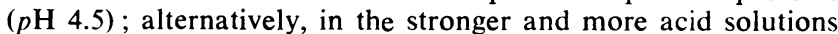
the serum proteins behave as an alkali and are precipitated as sulphates, being linked to the sulphuric acid radical dissociated from neutral proflavine sulphate.

Now we can appreciate what occurs in wounds treated with amino-acridine powders. Serous, sero-sanguineous, and purulent exudates protect underlying tissues and granulations from coagulation necrosis (the protein-precipitating action of proflavine sulphate B.P. and neutral proflavine sulphate) by their proteins suffering precipitation-greatest when the water content is high enough to permit of solution of the sulphates to protein-precipitating concentration, and least, or absent, when the fluid (non-cellular) portion approximates serum in composition; for, it will be recalled, the powder dissolves slowly in serum without any precipitation. Thus, in one of our unsuccessful cases neutral proflavine sulphate powder was almost all recovered from a protein-proflavine "cast" of the discharging through-and-through sinus treated. When proflavine sulphate B.P. is applied to a freshly "debrided "wound some of the powder dissolves in the oozing blood and serum to a maximum concentration of $0.3 \%$; but much more dissolves in the tissue fluid, where, in lower protein and greater water and electrolyte content, it is more easily soluble-to $1 \%, 2 \%$, or more in the case of neutral proflavine sulphate and approximating $0.4 \%$ to $0.6 \%$ when proflavine sulphate $B . P$. is used. Being thus above the critical levels of $0.5 \%$ (neutral proflavine sulphate) and $0.2 \%$ (proflavine sulphate B.P.), precipitation of the protein (coagulation necrosis) of the proximal tissue cells occurs to a depth of 2 to $4 \mathrm{~mm}$. Depending on the concentration of proflavine first attained, a certain amount of fine crystals of that substance also may be deposited. Clinically, in proportion to the amount of proflavine present to excess in wounds, an inflammatory reaction is set up, with capillary haemorrhages and oedema. This oedema occurred in many of our cases, and lasted for one to two days as a rule, although occasionally it took four days to subside. In the guinea-pig $50 \mathrm{mg}$. of neutral proflavine sulphate powder, suspended in saline and injected subcutaneously in the abdominal wall, resulted in local haemorrhagic necrosis of muscle and much inflammatory oedema of the loose areolar tissues ; while contiguous subjacent bowel wall became necrotic and the liver showed superficial haemorrhage and necrosis.

From our observations we cannot recommend the use of the sulphates of proflavine as pure powders in the treatment of wounds except under certain conditions. In this we are in agreement with a like statement of Russell and Falconer (1943), made as a result of their animal experiments with the three amino-acridines-proflavine sulphate B.P., 2:7-diaminoacridine, and 5-amino-acridine. A reason for the excellent results obtained by Mitchell and Buttle (1942) with proflavine sulphate B.P. in infected wounds of 5 to 12 days' standing is afforded by the "sparing" action of the purulent exudate present at that time and the fact that the infection was not yet "established" in bone. This is also true of similar cases recorded by Raven (1944). With neutral proflavine sulphate a like result is shown by our Case III. This is the only type of case in which it would be permissible to use the proflavines as powders, alone ; for if the dose were limited to 0.2 to $0.5 \mathrm{~g}$. for each application no added chemical inflammation or coagulation necrosis would be likely to occur. (There is no point in using sulphathiazole-proflavine powder in such cases. It would be of practically no additional value as a bacteriostatic, for the pus and tissue-breakdown products would surely neutralize the sulphathiazole.)

As regards the prophylactic use of the proflavine sulphates, we would suggest that for local application in powder form they be diluted with sulphathiazole to $0.5 \%$ in the case of neutral proflavine sulphate and $0.2 \%$ for proflavine sulphate B.P. The latter dilution is one-fifth of the concentration advocated by McIntosh and Selbie (1943) for the treatment of war wounds in general and of gas gangrene in particular ; but we suggest these concentrations as they are the highest possible of employ if coagulation necrosis is to be avoided and tissue damage kept to a minimum. By lowering the concentration of the proflavine sulphates to these levels their bactericidal and bacteriostatic values are practically not reduced, for the amino-acridines are rapidly bactericidal at a concentration of $0.2 \%$ (Garrod, 1940).

We have remarked already (Heggie et al., 1942) on the practical difficulty of ensuring the regular distribution, and the availability for solution, of sulphonamide powders in irregular wound cavities, to combat which we discarded the powder form in favour of a saline-glycerin-tragacanth sludge as a vehicle for sulphathiazole. Now in the case of neutral proflavine sulphate we employ a simple pouring tragacanth gel, containing a $0.5 \%$ saline solution of the drug, for use in the prophylactic treatment of wounds. The good results obtained to date may be attributed to the early stage at which compound fractures and flesh wounds have been received. Nevertheless, healing has not been impaired, bacteriostasis has been good, and we consider the use of this method worthy of further clinical trial. At most, $10 \mathrm{c} . \mathrm{cm}$., containing $0.05 \mathrm{~g}$. of neutral proflavine sulphate, has been used in any one wound at any one time.

\section{Summary and Conclusions}

Neutral proflavine sulphate has a place with the acid proflavine sulphate $B . P$. in the treatment of recently infected discharging wounds. When these amino-acridines are so used as pure powders, alone, and in small doses of from 0.2 to $0.5 \mathrm{~g}$. (repeated at 5 to 15 days' intervals, if. and as necessary), infection is reduced, discharges diminish and soon cease as the wound becomes dry, and healing proceeds. In such cases the proflavine sulphates produce little or no tissue necrosis, owing to the "sparing" action of the exudates, in which, after 
probably some measure of protein precipitation, they dissolve and diffuse to the tissues in effective bactericidal and bacteriostatic concentration.

When applied to freshly " debrided" wounds, or wounds with little exudate, a chemical inflammation occurs, attended by superficial coagulation necrosis to a depth of 2 to $4 \mathrm{~mm}$., due to solution of the sulphates in the tissue fluid to a proteinprecipitating concentration. Healing, at first arrested, is generally retarded, occurring under or after separation of the eschar. In saline solution the lowest concentration of these salts at which protein precipitation occurs is $0.6 \%$ and $0.25 \%$ of neutral proflavine sulphate monoacid and proflavine sulphate $B . P$. respectively. It is suggested, therefore, that the optimum concentration of these salts in a sulphathiazole-proflavine powder, for use prophylactically in war wounds, should be $0.5 \%$ for neutral proflavine sulphate and $0.2 \%$ for proflavine sulphate B.P., and, having regard to the difficulty of effectively applying powders to irregular wound cavities, saline-tragacanth gels of either of the proflavine sulphates, alone, and in the strengths mentioned, might be employed instead.

Our thanks are due to Col. F. Holmes, O.B.E., for his kind permission to carry out this investigation in the hospital under his command; and to Dr. R. P. Liston, of Imperial Chemical (Pharmaceuticals) Ltd., for providing the sample of neutral proflavine sulphate. To Major O. N. Bailey, R.A.M.C., surgical specialist at the hospital, and Capt. H. R. W. Lunt, R.A.M.C., graded orthopaedic surgeon to the hospital, our thanks are also due for the notes on the cases under their care and for their observations the notes on the
on the treatment.

REFERENCES

Albert, A., and Gledhill, W. (1943). Pharm. J., 151, 87.

Browning, C. H., Kennaway, E. L., Gulbransen, R., and Thornton, L. H. D. (1917). British Medical Journal, 1, 73

Garrod, L. P. (1940). Lancet, 1, 802 . Heggie, J. F., Kendall, A. W., and Heggie, R. M. (1942). British Medical Journal,

McIntosh, J., and Selbie, F. R. (1943). Lancet, 1, 793

Mitchell, G. A. G., and Buttle. G. A. H. (1942). Ibid., 2, 416.

Raven, R. W. (1944). Ibid., 2, 73.

Russel1, D. S., and Falconer, M. A. (1943). Ibid., 1, 580.

$=$

\section{HEREDITARY HAEMORRHAGIC TELANGIECTASIS}

\author{
D. CAPPON, M.R.C.S. \\ R.M.O., Dorset County Hospital
}

This comparatively rare disease is also known as Rendu-OslerWeber's disease or Goldstein's heredofamilial angiomatosis. It is defined as a hereditary, dystrophy of the capillaries, the disease being transmitted as a Mendelian dominant and affecting both sexes equally. The diagnosis is based on the following criteria: (1) it is hereditary and not sex-bound; (2) the presence of telangiectases or angiomatosis ; (3) the tendency to haemorrhage (Goldstein); to which a fourth may be added-the multiplicity of the lesions.

Although W. Legg first described a case in 1876, he presented it as a case of "haemophilia." Rendu in 1896 distinguished it from haemophilia, and Osler in 1901 described all its features. Parkes Weber described a family in 1907 and reviewed the literature in 1924. Goldstein (1932) made an extensive review of the literature, mentioning about 100 families (affecting 600 persons); but Hurst thought this review only caused confusion, since it included every kind of purpura and haemophilia and even a case of familial nephritis. Hurst and Plummer believed this condition to be more common than the number of cases on record would indicate; Price goes further in expressing the belief that it may be as common as haemophilia. The disease should not be diagnosed unless the patient shows multiple telangiectases with haemorrhage and comes from a family one or more members of which are known to have been affected in the past (Hurst and Plummer). Levy in 1933 stated that there were 320 known cases, all familial, though sporadic forms may arise. Hurst and Plummer (1932), Hampson (1932), and Yates (1932) made valuable contributions by reporting complete family histories of this condition. More recently (in 1944), while the case recorded below was being studied with a view to publication, since it shows unusual features, Campbell published a very similar case, and, following this, Wolfsohn added yet a further case to the literature.

\section{Case Record}

Mrs. C., aged 31, was admitted to hospital for a dilatation and curettage for incomplete abortion. Apart from this she wanted her blood condition investigated as she felt worried about it. The history was that since the age of 10 she had suffered from epistaxis, which soon became frequent and uncontrollable. She bled more from the right nostril than the left. Bleeding would start spontaneously at any time, day or night. It increased in frequency from once fortnightly to nearly every alternate day. It formerly ceased spontaneously, but now she has to control it by application of cold water and finger pressure. In some severe attacks she "bled white," having lost 1 to $1 \frac{1}{2}$ pints (her estimate) in the course of $1 \frac{1}{2}$ to 2 hours, which was the longest time bleeding continued. As a result she was always anaemic as a girl, suffering from lassitude and periodic swelling of the ankles, which prevented her from carrying out ordinary household duties. When free from attacks for a more prolonged period-i.e., two or three weeks-a strikingly high colouring of the cheeks had been observed. In such an instance her blood picture was: Hb, $112 \%$; R.B.C.s, 6,250,000; C.I., 0.9; W.B.C.s, 7,000 ; and on the strength of this and the history of bleeding she was diagnosed as a possible case of " polycythaemia " and told to avoid red meat, and the iron which she was taking regularly was discontinued. She also complained of headaches, which started at the age of 10. Often these headaches, which were parietal and frontal, preceded the epistaxis, and the only thing which seemed to make them better was a "good nose-bleed." She never had vomiting, fortification spectra, or blurring of vision. At about the same age she noticed that some red spots had appeared on her face. The first was on the right upper lip, then next on the tongue and cheeks and in the mouth. Lately she had discovered a few on the ends of her fingers. She has never bled from these spots. In the family history we find that her father had similar spots all over his face and fingers. One such bigger spot used to bleed. He also had severe headaches preceding epistaxis; the latter came from spidery naevi on both sides of the nasal septum. His case was regarded as thrombocytopenic purpura, but no blood reports are available. Transfusion was considered, though his heart condition was thought to make this too hazardous a procedure, so it was not performed. These symptoms increased in severity in middle life, and he died at 66 from severe loss of blood and mitral stenosis.

Further details of the family history are shown in the accompanying chart. Nearly all had epistaxis and telangiectasis, but a history of headaches could not be ascertained.

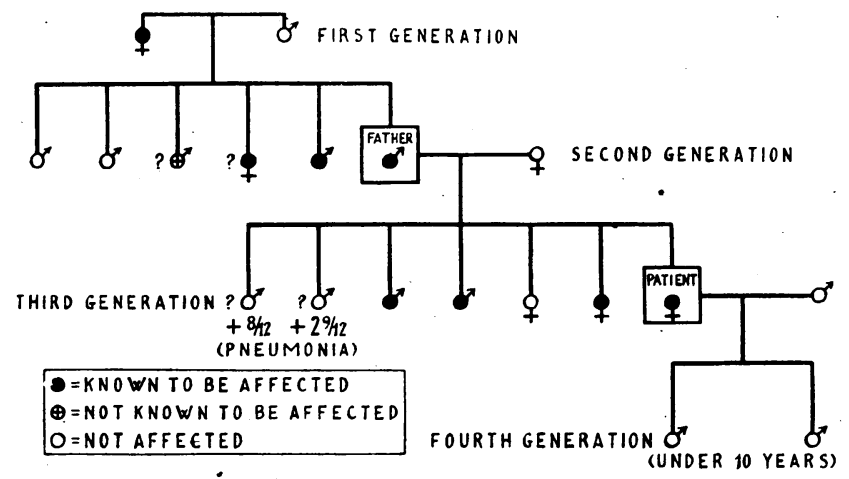

The obstetric history is that the patient has two live children and there have been two miscarriages. Menses are regular; there is no excessive loss. On the contrary, the tendency is to lose less now than formerly. Tonsillectomy was performed at 18, after which she had violent bleeding. There is no history of haemorrhage from anywhere else. A careful examination of the patient was carried out 8 weeks after her dilatation and curettage. Signs of anaemia were present-e.g., pale conjunctivae, lips, and koilonychia. Multiple asymmetrical telangiectases were present, mostly distributed over the upper lip and tip of the tongue, and on the cheeks; they were flat round spots with a red centre and paler periphery. It was remarkable that their colour disappeared, diminished, or remained unchanged on vitropression, according to their size, intensity of colour, and situation. Thus the very dark ones and the larger spots on the cheeks would be unaffected, but the smaller spots on the lips and tongue would disappear. This confirms the finding of Aubertin et al. (1933). The blood pressure was 130/70, and Hess's test was negative. Anterior rhinoscopy revealed a spidery naevus $1 \frac{1}{2}$ in. by $1 / 4$ in. on the right septum about 2 in. from the external nares. This had a scab on it, and bled with the slightest trauma. Several other spots were present on the left anterior turbinate and in the hypopharynx. The liver and spleen were impalpable. The cardiorespiratory system showed nothing of note. The blood picture (after 